\title{
BMJ Open Multimorbidity of cardiometabolic diseases: prevalence and risk for mortality from one million Chinese adults in a longitudinal cohort study
}

\author{
Dudan Zhang, ${ }^{\odot 1}$ Xun Tang, ${ }^{\odot 1}$ Peng Shen, ${ }^{2}$ Yaqin Si, ${ }^{1}$ Xiaofei Liu, ${ }^{1}$ Zhe Xu, ${ }^{1}$ \\ Jinguo Wu, ${ }^{3}$ Jingyi Zhang, ${ }^{3}$ Ping Lu, ${ }^{3}$ Hongbo Lin, ${ }^{2}$ Pei Gao ${ }^{1,4}$
}

To cite: Zhang D, Tang $X$, Shen $\mathrm{P}$, et al. Multimorbidity of cardiometabolic diseases: prevalence and risk for mortality from one million Chinese adults in a longitudinal cohort study. BMJ Open 2019;9:e024476. doi:10.1136/ bmjopen-2018-024476

- Prepublication history and additional material for this paper are available online. To view these files, please visit the journal online (http://dx.doi org/10.1136/bmjopen-2018024476).

DZ and XT contributed equally.

Received 28 May 2018

Revised 23 November 2018

Accepted 7 January 2019

Check for updates

(C) Author(s) (or their employer(s)) 2019. Re-use permitted under CC BY-NC. No commercial re-use. See rights and permissions. Published by BMJ.

${ }^{1}$ Department of Epidemiology and Biostatistics, School of Public Health, Peking University, Beijing, China

${ }^{2}$ Yinzhou District Center for Disease Control and Prevention, Ningbo, China

${ }^{3}$ Wonders Information Co., Ltd, Shanghai, China

${ }^{4}$ Key Laboratory of Molecular Cardiovascular Sciences (Peking University), Ministry of Education, Beijing, China

Correspondence to

Professor Pei Gao;

peigao@bjmu.edu.cn

\section{ABSTRACT}

Objectives The evolution of multimorbidity describes the continuum from a healthy status to the development of a single disease and further progression to multimorbidity with additional diseases. We investigated the evolution of cardiometabolic multimorbidity and risk for mortality in a Chinese population.

Design Longitudinal cohort study using data from the CHinese Electronic health Records Research in Yinzhou (CHERRY) study, with 5.43 million person-years follow-up (median 5.16 years).

Participants Data for 1038704 adults (total 22750 deaths) were analysed.

Exposure Cardiometabolic multimorbidity was defined as ever being diagnosed with two or more of three diseases: hypertension, diabetes and cardiovascular disease (CVD). Primary and secondary outcome measures Ageadjusted and sex-adjusted HRs were calculated for allcause mortality.

Results The cardiometabolic disease status of 105209 (10.1\%) individuals changed during the follow-up. The prevalence of cardiometabolic multimorbidity increased from $2.41 \%$ (95\% Cl: $2.38 \%$ to $2.44 \%$ ) to $5.94 \%$ (95\% Cl: $5.90 \%$ to $5.99 \%)$. Baseline multimorbidity status showed the $\mathrm{HR}(95 \% \mathrm{Cl})$ was 1.37 (1.33 to 1.42) in those with one disease, 1.71 (1.64 to 1.79) in those with two diseases and 2.22 (2.00 to 2.46) in those with three diseases. The highest HRs were observed for CVD only $(3.31,95 \% \mathrm{Cl}$ : 3.05 to 3.59$)$ or diabetes and CVD $(3.12,95 \% \mathrm{Cl}: 2.37$ to 4.11). Those with hypertension only had the lowest HR $(1.26,95 \% \mathrm{Cl}: 1.22$ to 1.30$)$. Longitudinal data showed the HRs $(95 \% \mathrm{Cl})$ in patients with one, two and three diseases were 1.36 (1.32 to 1.41), 2.03 (1.96 to 2.10) and 2.16 (2.05 to 2.29), respectively.

Conclusions The prevalence of cardiometabolic multimorbidity in a general Chinese population increased more than doubled over 5 years, indicating rapid evolution of cardiometabolic multimorbidity. A history of CVD dominates the risk for mortality. A complementary strategy for primary and secondary prevention of cardiometabolic diseases is needed in China.

\section{INTRODUCTION}

Considerable improvements in medical interventions and healthcare management mean
Strengths and limitations of this study

- This study is among the first to investigate the evolution of cardiometabolic multimorbidity and risk for mortality in a general population in China.

- We used a longitudinal design with continuous surveillance of cardiometabolic disease status to provide a better understanding of aetiological associations and causality, in addition to using multimorbidity assessed only at baseline.

- The CHinese Electronic health Records Research in Yinzhou (CHERRY) study is a large-scale natural population-based observational cohort study linking large data from integrated individual-level electronic health records; the study protocol of which was published in BMJ Open.

- The CHERRY study involves a regional cohort located in a developed area of China, and is, therefore, not nationally representative.

- This study had a relatively short follow-up period.

population ageing has become a major public health concern worldwide. Cardiometabolic multimorbidity is defined as the co-existence of two or more of three cardiometabolic disorders: hypertension, diabetes mellitus and cardiovascular disease (CVD). Cardiometabolic multimorbidity is becoming progressively more common globally, and the public health impact has received increased attention during the past several years. ${ }^{1-3}$ The individual prevalence of each disease is increasing rapidly in China, along with significant changes in economic development and lifestyles. ${ }^{4-7}$ Although some studies have evaluated the prevalence of multimorbidity in China using cross-sectional designs, ${ }^{8-11}$ they have been limited by factors, such as small sample sizes, high risk of bias according to the Strengthening the Reporting of Observational Studies in Epidemiology guideline, restriction to older adult populations $>60$ years) or including other chronic diseases (eg, 
chronic obstructive pulmonary disease or cancer) in the definition of multimorbidity. Moreover, multimorbidity is a continuum, covering the transition from healthy status to the development of a single disease and then progression to two or more diseases, with the possible addition of further diseases. ${ }^{12}$ The term 'evolution of multimorbidity' describes this process, which is generally evaluated from a longitudinal perspective. However, there have been limited studies on this topic, and none conducted among Chinese populations.

Multimorbidity is associated with high risk for mortality. ${ }^{13-15}$ The Emerging Risk Factors Collaboration (ERFC) reported that mortality was similarly associated with a history of diabetes, stroke or myocardial infarction (MI), and a multiplicative mortality risk was observed for any combination of these conditions in Western populations. ${ }^{16}$ However, hypertension was not included in that evaluation, and time-varying exposure information to update multimorbidity status was not available. A study using a longitudinal design with continuous surveillance of cardiometabolic disease status in a general population in addition to using multimorbidity assessed at baseline may provide a better understanding of aetiological associations and causality.

The CHinese Electronic health Records Research in Yinzhou (CHERRY) study is a longitudinal cohort study involving 1038704 adults (22750 deaths) from 2010 to 2016. Using CHERRY data, we aimed to provide reliable estimates about the prevalence of cardiometabolic comorbidities, investigate the evolution of multimorbidity during the follow-up and assess the association with mortality in a Chinese population.

\section{METHODS}

\section{Study design}

The CHERRY study is a longitudinal population-based ambispective cohort study focused on cardiovascular care and outcomes research. Individual participant data were extracted from the regional health information system in Yinzhou, an eastern coastal area of China. A detailed description of the data sources and the cohort profile was published previously. ${ }^{17}$ Specific data sources essential to the present study included: (1) the population census and registered health insurance database for individuals' general demographic characteristics; (2) health check databases, including health checks from the New Rural Cooperative Medical Scheme, older adults and adults with hypertension and diabetes; (3) inpatient and outpatient electronic medical records (EMRs); (4) disease surveillance and management database that captured the incidence of CVD, hypertension and diabetes (where cases were required to be reported for disease management by local general practitioners on confirmation of diagnosis) and (5) death certificates database where attribution of death refers to the primary cause provided by cause-specific mortality.
Individuals were included in the CHERRY study if they met all inclusion criteria: (1) aged $\geq 18$ years on 1 January 2009; (2) had complete information on date of birth and sex, and a valid healthcare identifier; (3) had been living in Yinzhou for at least 6 months and (4) had Chinese nationality. In total, 1053563 adults were originally enrolled in the CHERRY study. In this analysis, we choose 1 January 2010 as the date of inception to bypass the integration and preliminary test period of the EMR system and allow for better coverage of regional chronic disease management services. After excluding those who died in 2009 or entered the system after 2016, we included 1038704 participants in this study (online supplementary efigure 1). Follow-up in the health information system is generally continuous. CHERRY updates information for all cohort members annually from the health information system databases. Follow-up data through to 31 December 2016 were available for participants.

\section{Cardiometabolic multimorbidities and outcomes}

Cardiometabolic multimorbidity was defined as the presence of two or more of three diagnosed disorders: hypertension, diabetes or CVD (including coronary heart disease [CHD] and cerebrovascular diseases). We categorised participants into eight groups: (1) hypertension, (2) diabetes, (3) CVD, (4) hypertension and diabetes, (5) hypertension and CVD, (6) diabetes and CVD, (7) hypertension, diabetes and CVD, and (8) none of these diseases (reference group). Diagnosis of these cardiometabolic diseases (and date of diagnosis) were obtained from multiple sources: diseases management database (primary care), EMRs (hospital care) and disease surveillance database (disease registry). Date in disease surveillance was considered the gold standard for the date of diagnosis. Details of the comprehensive healthcare services for chronic disease surveillance and management provided in the study region were described previously. ${ }^{17}$ In addition to individuals' baseline cardiometabolic disease status, longitudinal information for the exposure was also used. To evaluate the association between mortality and a history of hypertension, diabetes, CVD and their cardiometabolic multimorbidities, changes in cardiometabolic disease status that occurred $\leq 30$ days before death were excluded, assuming that there was a direct connection between the change of cardiometabolic multimorbidity status and mortality within the acute phase. The primary outcome was all-cause mortality during the follow-up. Death was confirmed by the death certificate in the health information system, as previously described. ${ }^{18}$ Diseases and deaths were classified according to the International Classification of Diseases, 10th Revision.

\section{Statistical analysis}

Continuous and categorical baseline characteristics of participants were summarised by mean (SD) or numbers (percentage), respectively. Cardiometabolic multimorbidities were classified as eight combinations 
listed above. Numbers (and corresponding percentages) of participants who changed their cardiometabolic disease status during follow-up were summarised. Age- and sex-standardised prevalence and 95\% CI for cardiometabolic multimorbidity at baseline and the last visit were estimated for the overall population (and the population aged $\geq 40$ years in a supplementary analysis) based on the distribution of the 2010 Chinese population census. A Poisson regression model adjusted for sex was used to calculate mortality rates, adjusted for age 60 years. To investigate the association between cardiometabolic multimorbidity and mortality, we first assessed the associations between the cardiometabolic multimorbidity groups at baseline and risk for death from any cause. Furthermore, cardiometabolic multimorbidity status was modelled as a time-dependent exposure to enable updating of multimorbidity status during the follow-up. HRs and 95\% CI were calculated using Cox proportional hazard regression models stratified by sex and adjusted for age in the primary analyses of the overall population, the population aged $\geq 40$ years, and subgroups (sex, age, location [urban/rural], smoking status and body mass index [BMI] category). To explore the extent to which conventional factors (BMI, smoking, education level and location) explained the associations between cardiometabolic multimorbidity and mortality, HRs adjusted for these additional factors were calculated for people with full information on these factors. Finally, population attributable fractions (PAFs) and 95\% CI due to one, two or three cardiometabolic diseases were estimated by combining the proportional excess mortality $\left(\mathrm{X}_{1}, \mathrm{X}_{2}\right.$ and $\mathrm{X}_{3}$; where $\left.\mathrm{X}=\mathrm{HR}-1\right)$ and SE in each category with the corresponding prevalence at baseline $\left(\mathrm{P}_{1}, \mathrm{P}_{2}\right.$ and $\left.\mathrm{P}_{3}\right) .{ }^{19}$ The PAFs for one, two or three disorders are $\mathrm{P}_{1} \mathrm{X}_{1} / \mathrm{k}, \mathrm{P}_{2} \mathrm{X}_{2} / \mathrm{k}$ and $\mathrm{P}_{3} \mathrm{X}_{3} / \mathrm{k}$; where $\mathrm{k}=1+\mathrm{P}_{1} \mathrm{X}_{1}+\mathrm{P}_{2} \mathrm{X}_{2}+\mathrm{P}_{3} \mathrm{X}_{3}$. PAFs were calculated based on: (1) the prevalence and HRs of cardiometabolic multimorbidity at baseline and (2) the prevalence at last visit and HRs using time-variant cardiometabolic multimorbidity status. All p-values were two-tailed and not adjusted for multiple testing. We used Stata (version 14.0) for all data analyses, with a statistical significance level of $\mathrm{p}<0.05$.

\section{Patient and public involvement}

Patients were not involved in the development of the research question or measures, or the design, recruitment or conduct of this study. The results of this study will be disseminated to study participants and the public via this publication and the CHERRY study website (http:// www.cherry-study.org).

\section{RESULTS}

The present cohort was established using the procedures listed in online supplementary efigure 1 . We began with 1.28 million permanent residents in Yinzhou, China with a valid personal identifier. After excluding subjects
Table 1 Characteristics of participants at baseline

Participants $\geq \mathbf{1 8}$ Participants $\geq \mathbf{4 0}$ years years

\begin{tabular}{lll}
\hline Participants, $n$ & 1038704 & 545632 \\
Age (at baseline), mean & $42.51(14.84)$ & $53.58(11.63)$
\end{tabular}

(SD)

\begin{tabular}{|c|c|c|}
\hline $\begin{array}{l}\text { Body mass index }(\mathrm{kg} / \\
\left.\mathrm{m}^{2}\right) \text {, mean }(\mathrm{SD})\end{array}$ & $22.48(2.53)$ & $22.94(2.66)$ \\
\hline \multicolumn{3}{|c|}{ BMI (kg/m² Asian-specific cutoffs) } \\
\hline$<23$ (normal) & $562106(62.04)$ & $264283(53.65)$ \\
\hline $\begin{array}{l}23 \text { to }<25 \\
\text { (overweight) }\end{array}$ & $218019(24.06)$ & $134411(27.29)$ \\
\hline$\geq 25$ (obese) & $125862(13.89)$ & $93869(19.06)$ \\
\hline \multicolumn{3}{|l|}{ Gender, n (\%) } \\
\hline Male & 504525 (48.57) & $275382(50.47)$ \\
\hline Female & $534179(51.43)$ & $270250(49.53)$ \\
\hline \multicolumn{3}{|l|}{ Location, n (\%) } \\
\hline Urban & $316900(30.90)$ & $168208(31.18)$ \\
\hline Rural & $708642(69.10)$ & $371311(68.82)$ \\
\hline \multicolumn{3}{|l|}{ Education levels, n (\%) } \\
\hline $\begin{array}{l}\text { Primary school or } \\
\text { lower }\end{array}$ & $249881(29.11)$ & $219934(45.78)$ \\
\hline Middle school & $547890(63.83)$ & $248425(51.71)$ \\
\hline College or higher & $60642(7.06)$ & $12046(2.51)$ \\
\hline \multicolumn{3}{|c|}{ Smoking status (current), n (\%) } \\
\hline Never & $726241(78.87)$ & $388223(77.15)$ \\
\hline Former smoker & $20524(2.23)$ & $17678(3.51)$ \\
\hline Current smoker & 174084 (18.90) & $97328(19.34)$ \\
\hline
\end{tabular}

younger than 18 years old as at January 1, 2010, those that died before that date and those entered into the system after December 31, 2016, the present analyses included 1038704 CHERRY study participants aged $\geq 18$ years. Table 1 presents participants' baseline characteristics. Among all participants, $1025542(98.7 \%)$ had information on living location, $858413(82.6 \%)$ had information on education level, $905987(87.2 \%)$ had at least one BMI measurement and $920849(88.7 \%)$ had at least one smoking status measurement. The mean \pm SD age at baseline was $42.5 \pm 14.8$ years $(51.4 \%$ women). The mean BMI was $22.5 \pm 2.5 \mathrm{~kg} / \mathrm{m}^{2}$. According to Asian-specific BMI cutoffs, $24.1 \%$ were overweight $\left(23-25 \mathrm{~kg} / \mathrm{m}^{2}\right)$ and $13.9 \%$ were obese $\left(\mathrm{BMI} \geq 25 \mathrm{~kg} / \mathrm{m}^{2}\right)$. At the last visit during the follow-up, the mean BMI was $22.5 \pm 2.6 \mathrm{~kg} / \mathrm{m}^{2} ; 23.9 \%$ were overweight and $14.8 \%$ were obese. From baseline to the last visit, the proportion of former smokers changed from $2.2 \%$ to $2.4 \%$ and current smokers changed from $18.9 \%$ to $20.0 \%$.

\section{Prevalence and evolution of cardiometabolic multimorbidity}

At baseline, 85684 participants had one diagnosed cardiometabolic disease, 22871 had two diseases and 2203 had three diseases. The standardised prevalence rates were $9.32 \%$ (95\% CI: $9.26 \%$ to $9.37 \%$ ) for 
Table 2 Standardised prevalence of cardiometabolic disease status at baseline

\begin{tabular}{|c|c|c|c|c|c|c|}
\hline & \multicolumn{2}{|c|}{ Overall $(n=1038704)$} & \multicolumn{2}{|c|}{ Male $(n=504525)$} & \multicolumn{2}{|c|}{ Female $(n=534179)$} \\
\hline & No of cases & $\%(95 \% \mathrm{Cl})$ & No of cases & $\%(95 \% \mathrm{Cl})$ & No of cases & $\%(95 \% \mathrm{Cl})$ \\
\hline One disease & 85684 & 9.32 (9.26 to 9.37$)$ & 40883 & 8.64 (8.57 to 8.72 ) & 44801 & 10.01 (9.93 to 10.09$)$ \\
\hline DM & 14127 & 1.49 (1.46 to 1.51$)$ & 6992 & 1.43 (1.40 to 1.47$)$ & 7135 & 1.54 (1.51 to 1.58$)$ \\
\hline CVD & 2151 & 0.23 (0.22 to 0.24$)$ & 1194 & 0.25 (0.24 to 0.27$)$ & 957 & 0.21 (0.20 to 0.23$)$ \\
\hline $\mathrm{HT}+\mathrm{DM}$ & 11073 & 1.26 (1.24 to 1.29$)$ & 4722 & 1.03 (1.00 to 1.06$)$ & 6351 & 1.50 (1.46 to 1.53$)$ \\
\hline $\mathrm{HT}+\mathrm{CVD}$ & 11540 & 1.28 (1.25 to 1.30$)$ & 5481 & $1.18(1.15$ to 1.21$)$ & 6059 & $1.38(1.34$ to 1.41$)$ \\
\hline $\mathrm{DM}+\mathrm{CVD}$ & 258 & 0.03 (0.02 to 0.03$)$ & 146 & 0.03 (0.03 to 0.04$)$ & 112 & 0.03 (0.02 to 0.03$)$ \\
\hline Three diseases & 2203 & $0.26(0.25$ to 0.27$)$ & 841 & $0.19(0.17$ to 0.20$)$ & 1362 & $0.33(0.31$ to 0.35$)$ \\
\hline
\end{tabular}

CVD, cardiovascular disease; DM, diabetes mellitus; HT, hypertension.

one disease, $2.57 \%$ (95\% CI: $2.54 \%$ to $2.60 \%$ ) for two diseases and $0.26 \%$ (95\% CI: $0.25 \%$ to $0.27 \%$ ) for three diseases (table 2). The estimated prevalence of diagnosed cardiometabolic multimorbidity increased with age and was higher in women than men (figure 1). Among the population aged $\geq 40$ years, the standardised prevalence rates were $16.82 \%$ (95\% CI: $16.72 \%$ to $16.92 \%$ ) for one disease, $4.69 \%$ (95\% CI: $4.65 \%$ to $4.75 \%$ ) for two diseases and $0.47 \%$ (95\% CI: $0.45 \%$ to $0.49 \%$ ) for three diseases (online supplementary etable 1 ). In the population aged $\geq 60$ years, the prevalence rates increased to $31.90 \%$ (95\% CI: $31.65 \%$ to $32.14 \%$ ), $10.41 \%$ (95\% CI: $10.25 \%$ to $10.57 \%$ ) and $1.20 \%$ (95\% CI: $1.14 \%$ to $1.26 \%$ ) for one, two, and three diseases, respectively (online supplementary etable 2).

In total, 105209 participants $(10.1 \%)$ changed their cardiometabolic multimorbidity status during the (median) 5years follow-up (online supplementary etable 3). Regarding the history of CVD, 50458 (94.4\%) of all 53473 patients have information on the type of CVD. Within these patients, 26282 had CHD, 23538 had cerebrovascular diseases and 638 had both CHD

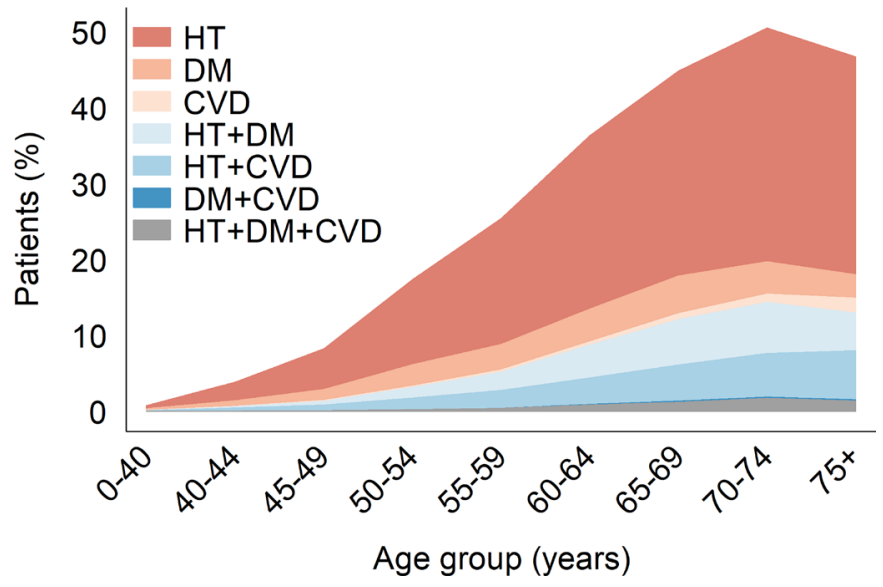

Figure 1 Prevalence of cardiometabolic disease according to age groups. CVD, cardiovascular disease; DM, diabetes mellitus; HT, hypertension. and cerebrovascular diseases. The crude prevalence of cardiometabolic multimorbidity increased from $2.41 \%$ (95\% CI: $2.38 \%$ to $2.44 \%$ ) to $5.94 \%$ (95\% CI: $5.90 \%$ to $5.99 \%$ ) (figure 2 \& online supplementary etable 3 ). Four disease groups (72104 participants; 68.5\%) lead all transitions during the follow-up: 47903 healthy subjects developed hypertension only; 8388 healthy subjects developed diabetes only; 9279 patients with hypertension developed CVD and 6534 patients with hypertension and diabetes

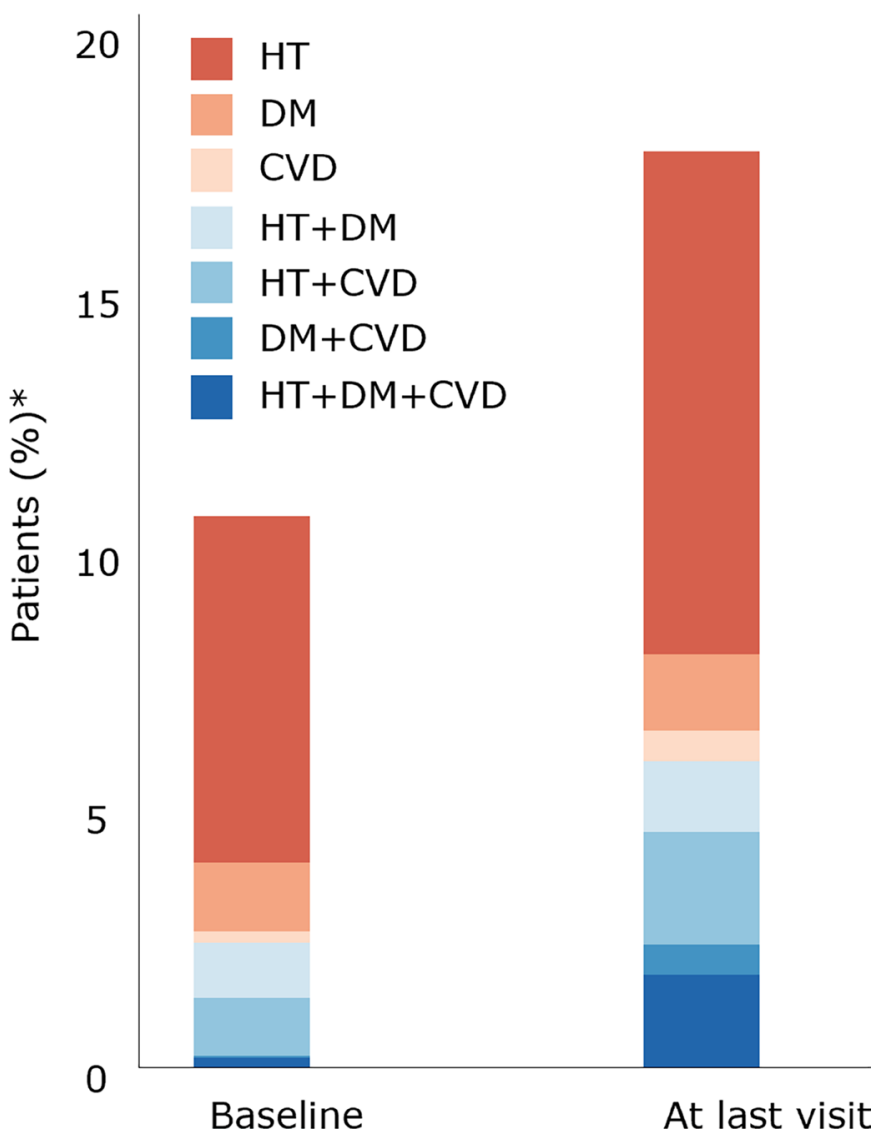

Figure 2 Evolution of cardiometabolic disease status during follow-up. Note: ${ }^{\star}$ Crude prevalence. CVD, cardiovascular disease; DM, diabetes mellitus; HT, hypertension. 


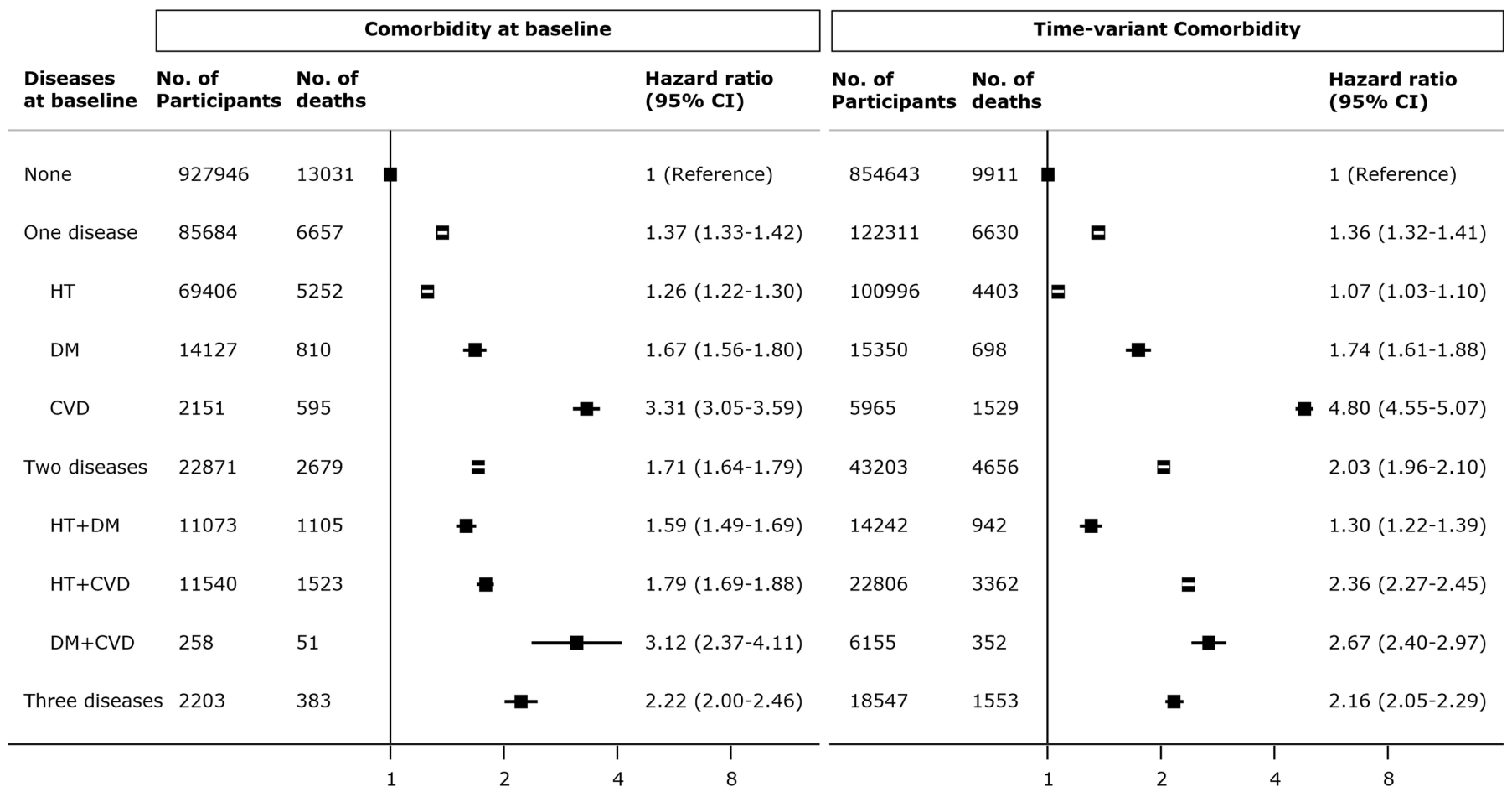

Figure 3 Age-adjusted and sex-adjusted HRs (95\%) for all-cause mortality by cardiometabolic disease status. CVD, cardiovascular disease; DM, diabetes mellitus; HT, hypertension.

developed CVD. Among 927946 participants without any diagnosed cardiometabolic disease at baseline, $73303(7.9 \%)$ developed one or more cardiometabolic diseases, of which 47903 (5.2\%) developed hypertension only. Among 85684 participants with one disease at baseline, $24041(28.1 \%)$ developed additional diseases. Of 69406 participants with hypertension at baseline, 12711 (18.31\%) had an incidence of CVD during the follow-up. Of 14127 participants with diabetes at baseline, 5386 (38.13\%) had an incidence of CVD during the follow-up. Finally, $7865(34.39 \%)$ of 22871 participants with two diseases developed all three diseases during the follow-up. The number of participants with all three diseases increased from 2203 at baseline to 18547 by the end of the study (online supplementary etable 3 ).

\section{Associations with mortality}

There were 22750 deaths during the 5.43 million personyears of follow-up (median follow-up time, 5.16 years). The sex-adjusted all-cause mortality rate at age 60 years was 5.68 per 1000 person-years (6.50 in men and 4.91 in women). In the analysis using baseline cardiometabolic multimorbidity status, the age- and sex-adjusted HRs for mortality were 1.37 (95\% CI: 1.33 to 1.42) for one disease, 1.71 (95\% CI: 1.64 to 1.79 ) for two diseases and 2.22 (95\% CI: 2.00 to 2.46) for three diseases, compared with participants without any disease (figure 3). The highest HRs were observed in patients with either a history of CVD only (3.31, 95\% CI: 3.05 to 3.59) or diabetes and CVD (3.12, 95\% CI: 2.37 to 4.11$)$. Patients with hypertension only had lowest HR (1.26, 95\% CI: 1.22 to 1.30). HRs for cardiometabolic multimorbidity were broadly similar in men and women, higher in younger participants (aged $<40$ years) and higher in those living in urban areas (online supplementary etable 4 ). The result of sensitivity analysis using baseline status excluding the initial 1 year of follow-up was broadly similar (online supplementary efigure 5).

The longitudinal data for cardiometabolic multimorbidity showed the age- and sex-adjusted HRs for mortality were broadly similar in those with one disease (1.36, 95\% CI 1.32 to 1.41), higher in those with two diseases (2.03, 95\% CI 1.96 to 2.10$)$, and similar in those with all three diseases (2.16, 95\% CI 2.05 to 2.29) compared with participants without any disease at the last visit (figure 3). Although the pattern of HRs within each multimorbidity combination was broadly similar to the analysis using baseline status, the HR in participants with hypertension only reduced to 1.07 (95\% CI: 1.03 to 1.10), whereas that for those with a history of CVD increased to $4.80(95 \%$ CI: 4.55 to 5.07). The HR in those with hypertension and diabetes was attenuated to 1.30 (95\% CI: 1.22 to 1.39), whereas that for participants with hypertension and CVD increased to 2.36 (95\% CI: 2.27 to 2.45). Broadly similar association were observed among the 788703 participants with full information on additional risk factors (BMI, smoking, education level and location) after further adjustment for those factors (online supplementary etable 6).

\section{Population attributable fractions}

Using the prevalence and the HRs for cardiometabolic disease status at baseline, the PAFs for all-cause mortality in the overall population aged $\geq 18$ years due to one, two 
Table 3 Population attributable fractions for cardiometabolic multimorbidity

Multimorbidity, \% $(95 \% \mathrm{Cl})$

\begin{tabular}{llll}
\hline One disease & Two diseases & Three diseases
\end{tabular}

\begin{tabular}{|c|c|c|c|c|}
\hline \multicolumn{5}{|c|}{ Using the prevalence and the HRs of cardiometabolic multimorbidity at baseline } \\
\hline Participants $\geq 18$ years & 3.30 (2.95 to 3.65$)$ & $1.73(1.56$ to 1.90$)$ & $0.30(0.25$ to 0.36$)$ & 5.34 (4.96 to 5.72$)$ \\
\hline Men & $3.10(2.65$ to 3.54$)$ & $1.45(1.24$ to 1.65$)$ & $0.18(0.12$ to 0.24$)$ & $4.72(4.24$ to 5.21$)$ \\
\hline Participants $\geq 40$ years & $5.10(4.52$ to 5.68$)$ & 2.85 (2.56 to 3.14$)$ & $0.50(0.40$ to 0.59$)$ & 8.44 (7.82 to 9.07$)$ \\
\hline Participants $\geq 60$ years & $6.11(5.07$ to 7.16$)$ & $4.60(4.01$ to 5.18$)$ & $0.96(0.75$ to 1.17$)$ & $11.67(10.54$ to 12.81$)$ \\
\hline Participants $\geq 18$ years & 4.17 (3.70 to 4.63$)$ & 4.36 (4.07 to 4.65$)$ & 2.13 (1.92 to 2.35$)$ & $10.66(10.11$ to 11.21$)$ \\
\hline Men & 4.19 (3.57 to 4.82 ) & 4.14 (3.76 to 4.52 ) & 1.69 (1.43 to 1.94$)$ & 10.02 (9.30 to 10.75$)$ \\
\hline Women & 4.33 (3.62 to 5.05 ) & 4.75 (4.30 to 5.20$)$ & 2.71 (2.35 to 3.07 ) & 11.80 (10.95 to 12.64$)$ \\
\hline Participants $\geq 40$ years & 5.92 (5.18 to 6.65$)$ & 6.95 (6.48 to 7.41$)$ & 3.49 (3.13 to 3.84$)$ & $16.35(15.52$ to 17.18$)$ \\
\hline Participants $\geq 60$ years & 6.47 (5.36 to 7.58$)$ & 11.03 (10.19 to 11.88$)$ & 5.75 (5.09 to 6.41$)$ & 23.25 (21.98 to 24.53$)$ \\
\hline
\end{tabular}

and three cardiometabolic diseases were $3.30 \%$ (95\% CI: $2.95 \%$ to $3.65 \%$ ), $1.73 \%$ (95\% CI: $1.56 \%$ to $1.90 \%$ ) and $0.30 \%$ (95\% CI: $0.25 \%$ to $0.36 \%$ ), respectively (table 3 ). In people aged $\geq 40$ years, the PAF increased to $5.10 \%$ (95\% CI: $4.52 \%$ to $5.68 \%$ ) for one disease, $2.85 \%(95 \%$ CI: $2.56 \%$ to $3.14 \%$ ) for two diseases and $0.50 \%$ (95\% CI: $0.40 \%$ to $0.59 \%$ ) for three diseases. Using the prevalence at the last visit and the HRs for time-variant cardiometabolic disease status, PAFs were $4.17 \%$ (95\% CI: $3.70 \%$ to $4.63 \%$ ) for one disease, $4.36 \%$ (95\% CI: $4.07 \%$ to $4.65 \%$ ) for two diseases and $2.13 \%$ (95\% CI: $1.92 \%$ to $2.35 \%$ ) for three diseases. The overall PAF increased from 5.34\% (95\% CI: $4.96 \%$ to $5.72 \%$ ) to $10.66 \%$ (95\% CI: $10.11 \%$ to $11.21 \%)$.

\section{DISCUSSION}

Our analyses of more than onemillion Chinese adults (22750 deaths during the follow-up) in a longitudinal cohort provided estimates of the prevalence of cardiometabolic multimorbidity (hypertension, diabetes and CVD) in a general population under real-world circumstances. This study also described the evolution of cardiometabolic diseases in this population over 5 years and evaluated associations with risk for all-cause mortality. Each of our main findings has potential implications.

First, $12.2 \%$ of Chinese adults aged $\geq 18$ years in a realworld general population had at least one diagnosed cardiometabolic disease, and nearly $3 \%$ had cardiometabolic multimorbidity. Hypertension was the dominant diagnosis in the group with one disease. The prevalence of multimorbidity at baseline increased with age, and was $5.2 \%$ and $11.6 \%$ in the population aged $\geq 40$ and $\geq 60$ years, respectively. One in four patients with any cardiometabolic disease had multimorbidity. This proportion was consistent in different age groups. Moreover, the proportion of patients with multimorbidity increased more than doubled during the (median)
5 years follow-up. The proportion of patients with all three diseases increased to ninefold from the baseline prevalence $(0.2 \%$ to $1.8 \%)$. Nearly $60 \%$ of patients with both hypertension and diabetes at baseline had an incidence of CVD during the follow-up. Over $50 \%$ of patients with diabetes and $20 \%$ of patients with hypertension developed cardiometabolic multimorbidity. These findings highlighted the rapid progression of cardiometabolic diseases.

Limited publications are available on the epidemiology of multimorbidity in a general Chinese population. A systematic review of nine published studies in China reported the prevalence of multimorbidity among those aged $\geq 60$ years ranged from $6.4 \%$ (95\% CI: 5.1 to 8.0$)$ to $76.5 \%$ (95\% CI: 73.6 to 79.2 ) ${ }^{10}$ However, most of the included studies considered morbidities in addition to cardiometabolic diseases and only reported prevalence based on the number of diseases, which prevented us from making direct comparisons. The estimated prevalence of single diagnosed diseases in our study was broadly consistent with estimates from national surveillance. For example, although the estimate of the prevalence of diabetes in China reached $10.9 \%$ in 2013, the national prevalence of diagnosed diabetes was only $4 \% .{ }^{4}$ Because our population was located in a developed area of China, our estimate of $3 \%$ is consistent with expectations, as the prevalence in this region is lower than the national estimate, even in traditional epidemiological surveys. Compared with developed countries, about $0.52 \%$ of participants aged $\geq 40$ years (mean age $53.6 \pm 11.6$ years) in our cohort had multimorbidity of diabetes and CVD (regardless of hypertension, $0.47 \%+0.05 \%=0.52 \%$, online supplementary etable 1 ), which was similar as $0.7 \%$ reported in the UK Biobank (mean age $56.7 \pm 8.1$ years). ${ }^{16}$ About $1.3 \%$ of participants aged $\geq 60$ years in our cohort had multimorbidity of diabetes and CVD, compared with $5 \%$ from a recent US survey involving people aged $\geq 65$ 
years. ${ }^{20}$ Previous studies in China and other countries have also shown that the prevalence of multimorbidity increased significantly with age. ${ }^{921}$ Older adults should, therefore, be a major population targeted for cardiometabolic multimorbidity prevention, considering population ageing.

Second, over the 5-year follow-up, we observed about a one-third higher mortality risk in patients with one condition at baseline, a two-thirds higher risk in patients with two diseases and just over a two-fold higher risk in patients with all three diseases. Although these results may suggest that associations between mortality and hypertension, diabetes and CVD were additive in this population, we found significant heterogeneities within each disease combination. Patients with only hypertension at baseline had the lowest HR (1.26) and those with CVD only at baseline had an HR of 3.31. This pattern was aggravated by applying longitudinal cardiometabolic disease status information. The HR of patients with hypertension only reduced to 1.07 and that of patients with GVD only increased to 4.8. Among around 70000 patients with hypertension at baseline, 15000 developed additional cardiometabolic diseases, and therefore, changed their disease status category. In addition, there were around $100000(10 \%)$ patients with hypertension in this study, and nearly half were identified during the follow-up (ie, suggesting that they were newly diagnosed). Therefore, the analysis using longitudinal disease status information showed that patients with hypertension had a lower HR than that using baseline status. In contrast, there was a small number of patients (just over 2000) with CVD only at baseline. Most patients with a history of CVD also had diabetes or hypertension. Around 4000 healthy participants at baseline developed CVD during follow-up. We speculated that the increment of the HR from 3.3 (baseline status) to 4.8 (longitudinal data) may be partly attributable to the relative short-term high risk after a first-ever CVD event. Similarly, we found an increased risk for those with both hypertension and CVD in the longitudinal analysis compared with that using baseline status.

Regardless of the existence of other diseases, a history of CVD tended to dominate risk for mortality, especially over the 5 years of follow-up. Among patients with any cardiometabolic disease, patients with CVD only had the highest risk, which may reflect a short-term effect of newly diagnosed CVD. Alternatively, it may also indicate that patients with other cardiometabolic diseases (eg, hypertension or diabetes) were likely to be more aware of their health condition. Moreover, among those with cardiometabolic multimorbidity, only patients with hypertension and diabetes had significantly lower HR, whereas other groups (ie, all with CVD) were broadly similar, even those with all three diseases.

Regarding the HRs for mortality from our study, we compared our findings with other studies. In ERFC study (involving 91 cohort studies, mainly Western populations) ${ }^{16}$ the estimated HR for mortality was about two in participants with one cardiometabolic multimorbidity condition (type 2 diabetes, CHD and stroke) and the association was multiplicative. However, they did not include hypertension and only used baseline disease status. We observed a broadly similar HR for patients with diabetes. The higher HR for patients with CVD (stroke or MI) in our population might be explained by differences in the overall healthcare services across countries with different economic levels. The estimated HR for patients with CVD and diabetes (regardless of hypertension) in our study was around 2.5, which was lower than the ERFC estimate (around 3.5-4), but consistent with the Hispanic Established Population for the Epidemiological Study of the Elderly (at 2.4) ${ }^{22}$ This may imply that ethnic disparity could explain the differences. We also showed that cardiometabolic multimorbidity had a more serious impact on those living in urban areas compared with rural areas. This suggests that it is important to consider location differences in developing strategies to improve public health.

The strength of this study was that it was a large-scale, comprehensive study under real-world circumstances. We used longitudinal data for cardiometabolic disease status to assess the prevalence and evolution of cardiometabolic multimorbidity during follow-up. However, this study also had several limitations. First, the CHERRY study is based on a regional population in a developed area of China and is not nationally representative. Second, this study had a relatively short follow-up period and the long-term effect of cardiometabolic multimorbidity needs to be further evaluated. Third, the accurate time of disease onset, especially hypertension and diabetes, were not available. We could only approximate this information using the first diagnosis time for these diseases. Information on medication use and risk control was also not available. Finally, our laboratory data sources included lipids measurements for only $25 \%-30 \%$ of the population, which prevented us from model adjustment for conventional lipids risk factors. However, the ERFC results showed broadly similar HRs for cardiometabolic multimorbidity and mortality after further adjustment for smoking, BMI, systolic blood pressure, high-density lipoprotein, total cholesterol, socioeconomic status and diet. $^{16}$

\section{CONCLUSIONS}

The prevalence of patients with cardiometabolic multimorbidity in a general population in China increased more than doubled over 5 years, indicating a rapid evolution of cardiometabolic multimorbidity. Among all combinations, history of CVD leads the risk for mortality. Our findings highlight the need for a complementary strategy for primary and secondary prevention of cardiometabolic diseases in China.

Acknowledgements The authors thank the Health and Family Planning Bureau of Yinzhou District for providing access to the administrative databases used in the study and Audrey Holmes, MA, from Liwen Bianji, Edanz Group China (https://www. liwenbianji.cn/) for editing the English text of a draft of this manuscript. 
Contributors DZ, XT and PG drafted the manuscript. DZ, XT, PS and PG conceived and designed the study. DZ, YS and HL made substantial contributions to the study design. XT, XL, PS and HL are responsible for study coordination; XT, PS, JW, JZ and $\mathrm{HL}$ are responsible for data quality control; DZ, PL and YS are responsible for data wrangling and $\mathrm{XT}, \mathrm{DZ}, \mathrm{YS}, \mathrm{XL}, \mathrm{ZX}$ and $\mathrm{PG}$ are responsible for data analysis. All authors contributed to the writing of the manuscript in an iterative manner, and have read and approved the final manuscript.

Funding This study is supported by the National Natural Science Foundation of China (91546120 and 81573226) and the National Thousand Talents Program for Distinguished Young Scholars, China (QNQR201501).

Competing interests None declared.

Patient consent for publication Not required.

Ethics approval This study was approved by the Peking University Institutional Review Board (IRB00001052-16011)

Provenance and peer review Not commissioned; externally peer reviewed.

Data sharing statement № additional data are available.

Open access This is an open access article distributed in accordance with the Creative Commons Attribution Non Commercial (CC BY-NC 4.0) license, which permits others to distribute, remix, adapt, build upon this work non-commercially, and license their derivative works on different terms, provided the original work is properly cited, appropriate credit is given, any changes made indicated, and the use is non-commercial. See: http://creativecommons.org/licenses/by-nc/4.0/.

\section{REFERENCES}

1. Tinetti ME, Fried TR, Boyd CM. Designing health care for the most common chronic condition-multimorbidity. JAMA 2012;307:2493-4.

2. Glynn LG. Multimorbidity: another key issue for cardiovascular medicine. Lancet 2009;374:1421-2

3. Barnett K, Mercer SW, Norbury M, et al. Epidemiology of multimorbidity and implications for health care, research, and medical education: a cross-sectional study. The Lancet 2012;380:37-43

4. Wang L, Gao P, Zhang M, et al. Prevalence and ethnic pattern of diabetes and prediabetes in China in 2013. JAMA 2017;317:2515-23.

5. Wang $W$, Jiang $B$, Sun $H$, et al. Prevalence, incidence, and mortality of stroke in china: Results from a nationwide population-based survey of 480687 adults. Circulation 2017;135:759-71.

6. Chen WW, Gao RL, Liu LS, et al. China cardiovascular diseases report 2015: a summary. J Geriatr Cardiol 2017;14:1-10.

7. Wu Y, Benjamin EJ, MacMahon S. Prevention and control of cardiovascular disease in the rapidly changing economy of China. Circulation 2016;133:2545-60.
8. Wang SB, D'Arcy C, Yu YQ, et al. Prevalence and patterns of multimorbidity in northeastern China: a cross-sectional study. Public Health 2015;129:1539-46.

9. Wang HH, Wang JJ, Wong SY, et al. Epidemiology of multimorbidity in China and implications for the healthcare system: cross-sectional survey among 162,464 community household residents in southern China. BMC Med 2014;12:188.

10. Hu X, Huang J, Lv Y, et al. Status of prevalence study on multimorbidity of chronic disease in China: systematic review. Geriatr Gerontol Int 2015;15:1-10.

11. Gu J, Chao J, Chen W, et al. Multimorbidity in the communitydwelling elderly in urban China. Arch Gerontol Geriatr 2017;68:62-7.

12. Ruel G, Lévesque JF, Stocks N, et al. Understanding the evolution of multimorbidity: evidences from the North West Adelaide Health Longitudinal Study (NWAHS). PLoS One 2014;9:e96291.

13. Bragg F, Holmes MV, lona A, et al. Association between diabetes and cause-specific mortality in rural and urban areas of China. JAMA 2017;317:280-9.

14. Yang G, Wang Y, Zeng Y, et al. Rapid health transition in china, 1990 2010: Findings from the global burden of disease study 2010. Lancet 2013;381:1987-2015.

15. Lewington S, Lacey $B$, Clarke $R$, et al. The burden of hypertension and associated risk for cardiovascular mortality in China. JAMA Intern Med 2016;176:524-32.

16. Di Angelantonio E, Kaptoge S, Wormser D, et al. Association of cardiometabolic multimorbidity with mortality. JAMA 2015;314:52-60.

17. Lin $\mathrm{H}$, Tang $\mathrm{X}$, Shen $\mathrm{P}$, et al. Using big data to improve cardiovascular care and outcomes in China: a protocol for the CHinese Electronic health Records Research in Yinzhou (CHERRY) Study. BMJ Open 2018;8:e019698.

18. Wang JB, Gu MJ, Shen P, et al. Body mass index and mortality: A 10-year prospective study in China. Sci Rep 2016;6:31609.

19. Global BMI Mortality Collaboration, Di Angelantonio E, Bhupathiraju $\mathrm{S}$, et al. Body-mass index and all-cause mortality: individualparticipant-data meta-analysis of 239 prospective studies in four continents. Lancet 2016;388:776-86.

20. Weiss $\mathrm{CO}$, Boyd $\mathrm{CM}, \mathrm{Yu} \mathrm{Q}$, et al. Patterns of prevalent major chronic disease among older adults in the United States. JAMA 2007;298:1158-2.

21. Barnett K, Mercer SW, Norbury M, et al. Epidemiology of multimorbidity and implications for health care, research, and medical education: a cross-sectional study. Lancet 2012;380:37-43.

22. Otiniano ME, Du XL, Ottenbacher K, et al. The effect of diabetes combined with stroke on disability, self-rated health, and mortality in older Mexican Americans: results from the Hispanic EPESE. Arch Phys Med Rehabil 2003;84:725-30. 